\title{
Efficacy of Different Leuprolide Administration Schedules in Premenopausal Breast Cancer: A Retrospective Review
}

\author{
Daniel C. Kendzierski, Pharm.D. \\ Bryan P. Schneider, M.D. \\ Patrick J. Kiel, Pharm.D., BCPS, BCOP \\ Indiana University Melvin and Bren Simon Cancer Center
}

535 Barnhill Dr, Indianapolis, IN 46202

Presented at Great Lakes Pharmacy Resident Conference, Purdue University, Lafayette, Indiana April $26^{\text {th }} 2017$

This is the author's manuscript of the article published in final edited form as:

Kendzierski, D. C., Schneider, B. P., \& Kiel, P. J. (2018). Efficacy of Different Leuprolide Administration Schedules in Premenopausal Breast Cancer: A Retrospective Review. Clinical Breast Cancer. https://doi.org/10.1016/j.clbc.2018.04.005 


\begin{abstract}
Purpose: Leuprolide is a safe and effective treatment of estrogen receptor positive, premenopausal breast cancer. Data from the SOFT/TEXT trials solidified leuprolide, in combination with an aromatase inhibitor, as an effective hormonal treatment for premenopausal breast cancer. However, the efficacy of monthly leuprolide depot compared to every threemonth leuprolide depot in combination with an aromatase inhibitor in premenopausal women with estrogen receptor positive breast cancer is unclear.
\end{abstract}

Patients and Methods: In this single center retrospective study, 201 patients were enrolled between Jan $1^{\text {st }}, 2015$, and Oct $1^{\text {st }}, 2016 ; 100$ were included in the leuprolide $7.5 \mathrm{mg}$ monthly injection plus aromatase inhibitor group and 101 in the leuprolide $22.5 \mathrm{mg}$ every 3 -month injection plus aromatase inhibitor group. The primary endpoint was the proportion of patients who achieved ovarian ablation, defined as an estradiol concentration less than $40 \mathrm{pg} / \mathrm{mL}$ and an FSH concentration of 23-116 mU/mL after 3 months of treatment. Significance threshold was a $\mathrm{P}<0.05$ (two-sided). Secondary endpoints included disease free survival and overall survival at one year follow-up, and adverse events reported during treatment.

Results: All patients in the leuprolide monthly arm achieved ovarian ablation compared to 100 (99\%) of the 101 patients in the every 3 -month $\operatorname{arm}(\mathrm{P}=1)$. The disease free survival rate at 1 year was $95 \%$ in the monthly leuprolide arm and $97 \%$ in the every 3 -month arm $(\mathrm{P}=0.75)$. The overall survival rate at 1 year was $100 \%$ in the leuprolide monthly arm and $99 \%$ in the every 3month arm $(\mathrm{P}=1)$. The most common treatment-related adverse events between the two groups were musculo-skeletal pain, hot flashes, fatigue, and insomnia.

Downloaded for Anonymous User (n/a) at Indiana University - Ruth Lilly Medical Library from ClinicalKey.com by Elsevier on April $27,2018$. For personal use only. No other uses without permission. Copyright $\odot 2018$. Elsevier Inc. All rights reserved. 
Conclusion: Leuprolide acetate depot administered every 3 months is as efficacious and tolerable as monthly injection, in combination with an aromatase inhibitor for patients with premenopausal, hormone receptor positive breast cancer.

\section{INTRODUCTION}

Leuprolide acetate Depot $\left(\right.$ Lupron $\left.^{\circledR}\right)$ is an intramuscular injection that is currently used off-label for premenopausal ovarian ablation in women with breast cancer who are estrogen receptor positive and on an aromatase inhibitor. It is an agonist of gonadotropin releasing hormone $(\mathrm{GnRH})$ and acts as a potent inhibitor of gonadotropin secretion. Continuous administration results in suppression of ovarian steroidogenesis due to decreased levels of luteinizing hormone ( $\mathrm{LH})$ and follicle stimulating hormone (FSH) with subsequent decrease in estrogen levels. ${ }^{1}$ It is available in many different dosages and administration schedules and also carries several FDA indications, including treatment of prostate cancer. However, leuprolide acetate currently does not carry an FDA approval for the treatment of breast cancer. Despite this, there have been several large, multicenter, randomized-controlled trials that have proven adjuvant treatment of estrogen receptor positive breast cancer in premenopausal woman with a GnRH analogue is an effective therapy. ${ }^{3-5}$

Based on the current literature, there has been no study to date evaluating different administration schedules of leuprolide acetate depot in combination with an aromatase inhibitor as adjuvant treatment in premenopausal, estrogen receptor-positive breast cancer. We report the results of a single institution experience comparing once monthly leuprolide depot to every three-month leuprolide depot in combination with an aromatase inhibitor in premenopausal women with estrogen receptor positive breast cancer. 


\section{METHODS}

\section{Patients}

Eligibility in the trial required documented premenopausal status. Inclusion criteria were histologically proven operable breast cancer, and tumor that expressed estrogen or progesterone receptors in at least $10 \%$ of the cells, as assessed with the use of immunohistochemical testing. All patients must have been 18 years of age or older, undergone either a total mastectomy with subsequent optional radiotherapy or breast-conserving surgery with subsequent radiotherapy, and received adjuvant ovarian suppression in combination with an aromatase inhibitor for at least 1 year. Women who underwent bilateral oophorectomy or ovarian irradiation were excluded.

\section{Design}

This study was designed as a retrospective chart review of patients identified from a Cerner generated report based on administration of leuprolide acetate in the infusion clinic at the Indiana University Melvin and Bren Simon Cancer Center and surrounding infusion centers in Indianapolis, Indiana. Patients were separated into two treatment arms based on a documented physician order and administration of: leuprolide acetate depot $7.5 \mathrm{mg}$ intramuscular injection monthly or leuprolide acetate depot $22.5 \mathrm{mg}$ intramuscular injection every 3 months.

The primary endpoint was the proportion of patients who achieved ovarian ablation, defined as an estradiol concentration of less than $40 \mathrm{pg} / \mathrm{mL}$ and an FSH concentration of 23-116 $\mathrm{mIU} / \mathrm{mL}$ after 3 months of treatment. Secondary endpoints included the following: disease free survival, defined as the time from receiving the first dose of leuprolide acetate depot to the appearance of invasive recurrence of breast cancer (local, regional, or distant), invasive contralateral breast cancer, second (nonbreast) invasive cancer, or death without breast cancer 
recurrence or second invasive cancer; overall survival, defined as the time from randomization to death from any cause; and safety, defined as the incidence of any grade adverse events reported while receiving treatment. For patients who did not have an end-point event, the times were censored at the date of a year after initial treatment date.

\section{Study Oversight}

The trial protocol was designed by the lead author and was approved by the institutional review board at the Indiana University Melvin and Bren Simon Cancer Center in Indianapolis, Indiana. The first author contributed to the collection of data, statistical analysis, and interpretation of the results. All the authors approved the final version of the manuscript, and assume responsibility for the adherence of the study protocol and for the accuracy and completeness of the data and analyses reported. No funder had any role in the design, conduct, analysis, or interpretation of the study or in the writing of this report.

\section{Statistical analysis}

The statistical analysis plan for this study is to compare the proportion patients who achieved ovarian ablation between the two groups with the hypothesis that there would be no difference. Differences in baseline characteristics were analyzed using the Pearson Chi-Square test for all categorical data and the student t-test for all continuous data. Kaplan-Meier estimates of time-to-event end points were calculated. Log-rank test was used to calculate P values comparing the two groups. All tests were two sided and a $\mathrm{P}<0.05$ was considered statistically significant. The statistical testing was performed using Statistical Package for Social Sciences Windows Version 21 (SPSS Inc., Chicago, IL, USA).

\section{RESULTS}




\section{Study Population}

From between Jan $1^{\text {st }}, 2015$, and Oct $1^{\text {st }}, 2016$ a total of 201 patients met eligibility criteria for the study; 100 patients in the leuprolide $7.5 \mathrm{mg}$ monthly arm and 101 patients in the leuprolide $22.5 \mathrm{mg}$ every 3 months arm. The characteristics at baseline of the patients who were included are shown in Table 1. The median age at first dose of adjuvant hormonal therapy was 40 years. Node positive disease was present in $37 \%$ of patients overall, and in $39 \%$ and $35 \%$ of the leuprolide monthly and every 3 month arms respectively. There was no significant difference between groups with respect to age, prior chemotherapy, tumor grade, lymph node involvement, tumor size, HER-2 status, or prior endocrine therapy. The cohort of patients who received prior endocrine therapy had been treated with tamoxifen to assess tolerance to hormonal therapy or as a bridge to a GnRH agonist plus aromatase inhibitor.

\section{Efficacy}

Three months after the first dose of leuprolide depot, all patients in the $7.5 \mathrm{mg}$ monthly group achieved ovarian ablation and all but one patient in the $22.5 \mathrm{mg}$ every 3 -month group achieved ovarian ablation $(\mathrm{P}=1)$. The distribution of estradiol and FSH levels between the 2 cohorts at three month follow-up are reported in Table 2. At 1 year follow-up, 9 patients had disease recurrence or second invasive disease or died. The rate of disease free survival at 1 year was $95 \%$ among patients who received leuprolide $7.5 \mathrm{mg}$ monthly as compared with $96 \%$ among those who received leuprolide $22.5 \mathrm{mg}$ every three months $(\mathrm{P}=0.75)$ (Figure 1). Death was reported in 1 patient in the leuprolide $22.5 \mathrm{mg}$ every 3 -months; the difference was not significant.

\section{Adverse Events}


After one year follow-up of all patients in this study, $95 \%$ of patients experienced one or more side effects related to treatment with either leuprolide acetate or the aromatase inhibitor. The most common side effect reported, per chart review, across both arms was muscolo-skeletal symptoms (90\%) such as arthralgia (Table 3). Many of the side effects related to the administration of leuprolide that were reported are hot flashes, insomnia, fatigue, depression, and vaginal dryness. These adverse effects were expected following the administration of combination hormonal therapy with leuprolide and an aromatase inhibitor based on previous reports. ${ }^{3-5}$

\section{DISCUSSION}

This study demonstrates that the leuprolide acetate $22.5 \mathrm{mg}$ intramuscular injection is as efficacious and tolerable as the monthly $7.5 \mathrm{mg}$ formulation, in combination with an aromatase inhibitor. Almost all patients, with the exception of one, achieved ovarian ablation in the two arms by month three of treatment. This is comparable to the original research by Boccardo and colleagues, who were the first to evaluate the endocrinological and clinical activity of a slowrelease formulation of leuprolide acetate in breast cancer patients. ${ }^{2}$ A total of 50 pre- or perimenopausal patients with early- or late-stage breast cancer who were candidates for endocrine treatment were included in the study and randomly allocated to receive either $3.75 \mathrm{mg}$ of leuprolide acetate every month or $11.25 \mathrm{mg}$ of leuprolide acetate every 3 months. In all, 23 patients were allocated to the monthly formulation and 27 , to the 3 -monthly formulation. There was no evidence of any difference in clinical outcome or drug-induced side effects, hot flushes being recorded in about $50 \%$ of patients in both groups. All endocrine parameters, particularly estradiol levels, were suppressed to a similar extent. These results are validated in our study, however different doses of leuprolide acetate depot were used and Boccardo's study did not use combination GnRH agonists with an aromatase inhibitor. Our 
institution uses the approved prostate cancer doses of leuprolide acetate depot instead of the offlabel doses used in the previous study. Previous large scale, randomized, controlled trials such as The TABLE Study have also used the lower dose of $11.25 \mathrm{mg}$ leuprolide every-3-months when studied in combination with tamoxifen versus chemotherapy plus tamoxifen. ${ }^{3}$ This leaves some area of uncertainty in terms of which dose of GnRH agonist is optimal as other trials such as the SOFT/TEXT trials utilized a different GnRH agonist in the form of triptorelin. Triptorelin was dosed in this study in accordance with the FDA approved prostate cancer dosing of $3.75 \mathrm{mg}$ intramuscularly every 28 days. $^{4,5}$

While there appears to be no difference in disease free survival, overall survival, or adverse events, these results must be interpreted with caution as our study was not randomized nor stratified for stage, chemotherapy received, age, grade, or HER2 status. The SOFT/TEXT trials solidified GnRH agonists as a definitive option in adjuvant therapy for premenopausal, estrogen receptor positive breast cancer. ${ }^{4,5}$ The primary analysis of the combined data tested the hypothesis that adjuvant therapy with an aromatase inhibitor, exemestane, improved disease-free survival, as compared with tamoxifen, among premenopausal women treated plus ovarian suppression in the form of monthly triptorelin. The results revealed that at 5 years, patients who received exemestane plus ovarian suppression has superior disease free survival and less recurrence compared to tamoxifen plus ovarian suppression. Because these results were published recently, it wasn't until 2 years ago we saw a shift in prescribing habits of leuprolide acetate depot at our institution. Therefore, we are unable to capture more long term survival data at 5 years, versus 1 year in our study, of follow-up at this time. Of note, the one patient who did not achieve ovarian ablation in the every 3-month leuprolide group did not have disease recurrence or die at 1 year follow-up. This patient, however, was only 28 years of age. In total, there were 7 patients across both groups who were less than 30 years of age. 
The retrospective design comes with inherent limitations. Our patient population was small and a single institutional experience compared to larger randomized, multicenter trials of adjuvant hormonal therapy in breast cancer patients. However, our study was powered to find a significant difference in the primary outcome. There were also some data points that were unable to be captured because we must rely on documentation in patient charts. Accuracy and documentation of adverse events such as injection site pain was unable to be collected because of this. A future randomized, prospective study is warranted to confirm the result of this study and to allow for a longer follow-up period.

In conclusion, leuprolide acetate depot administered every 3 months is as efficacious and tolerable as monthly injection, in combination with an aromatase inhibitor for patients with premenopausal, hormone receptor positive breast cancer. Prescribing leuprolide acetate depot $22.5 \mathrm{mg}$ intramuscularly every three months for patients may allow for less infusion chair time, cost savings, and higher patient satisfaction.

\section{REFERENCES}

1. Lupron Depot ${ }^{\circledR}$ [package insert]. AbbVie Inc. North Chicago, IL; 2013

2. Boccardo F, Rubagotti A, Amoroso D, et al. Endrocrinological and clinical evaluation of two depot formulations of leuprolide acetate in pre- and perimenopausal breast cancer patients. Cancer Chemother Pharmacol 1999;43:461-466

3. Schmid P, Untch M, Kosse V, et al. Leuprorelin Acetate Every-3-Months Depot Versus Cyclophosphamide, Methotrexate, and Fluorouracil As Adjuvant Treatment in Premenopausal Patients With Node-Positive Breast Cancer: The TABLE Study. J Clin Onc 2007;25:2509-15 
4. Pagani O, Regan MM, Walley BA, et al. Adjuvant Exemestane with Ovarian Suppression in Premenopausal Breast Cancer. N Engl J Med 2014;371:107-118

5. Francis P, Regan M, Fleming G, et al. Adjuvant Ovarian Suppression in Premenopausal Breast Cancer. N Engl J Med 2014;372:436-46 
Table 1.

\begin{tabular}{|c|c|c|c|}
\hline \multicolumn{4}{|c|}{ Baseline Characteristics } \\
\hline Characteristic & $\begin{array}{c}\text { Leuprolide Depot } \\
7.5 \mathrm{mg} \\
\mathrm{N}=100(\%)\end{array}$ & $\begin{array}{c}\text { Leuprolide Depot } \\
22.5 \mathrm{mg} \\
\mathrm{N}=101(\%)\end{array}$ & $P$ value \\
\hline Age (SD) & $39(7.2)$ & $40.1(7.7)$ & 0.33 \\
\hline $\begin{array}{l}\text { Prior Chemotherapy } \\
\text { Tumor grade }\end{array}$ & $53(53)$ & $53(52.5)$ & 0.94 \\
\hline Grade 1 & $27(27)$ & $25(24)$ & 0.91 \\
\hline Grade 2 & $47(47)$ & $49(48.5)$ & 0.83 \\
\hline Grade 3 & $25(25)$ & $24(23.8)$ & 0.84 \\
\hline Lymph node involvemen & & & \\
\hline 0 & $60(60)$ & $65(64.4)$ & 0.52 \\
\hline $1-3$ & $27(27)$ & $25(24.8)$ & 0.72 \\
\hline $4+$ & $12(12)$ & $10(9.9)$ & 0.63 \\
\hline Tumor size & & & \\
\hline$<1 \mathrm{~cm}$ & $19(19)$ & $16(15.8)$ & 0.56 \\
\hline $1-2 \mathrm{~cm}$ & $50(50)$ & $51(50.5)$ & 0.94 \\
\hline $2-5 \mathrm{~cm}$ & $28(28)$ & $28(27.7)$ & 0.97 \\
\hline$>5 \mathrm{~cm}$ & $2(2)$ & $5(5)$ & 0.45 \\
\hline HER-2 status & & & \\
\hline $\begin{array}{c}\text { Positive } \\
\text { Prior endocrine therapy }\end{array}$ & $14(14)$ & $16(15.8)$ & .71 \\
\hline Yes & $33(33)$ & $33(32.7)$ & 0.96 \\
\hline
\end{tabular}


Figure 1.

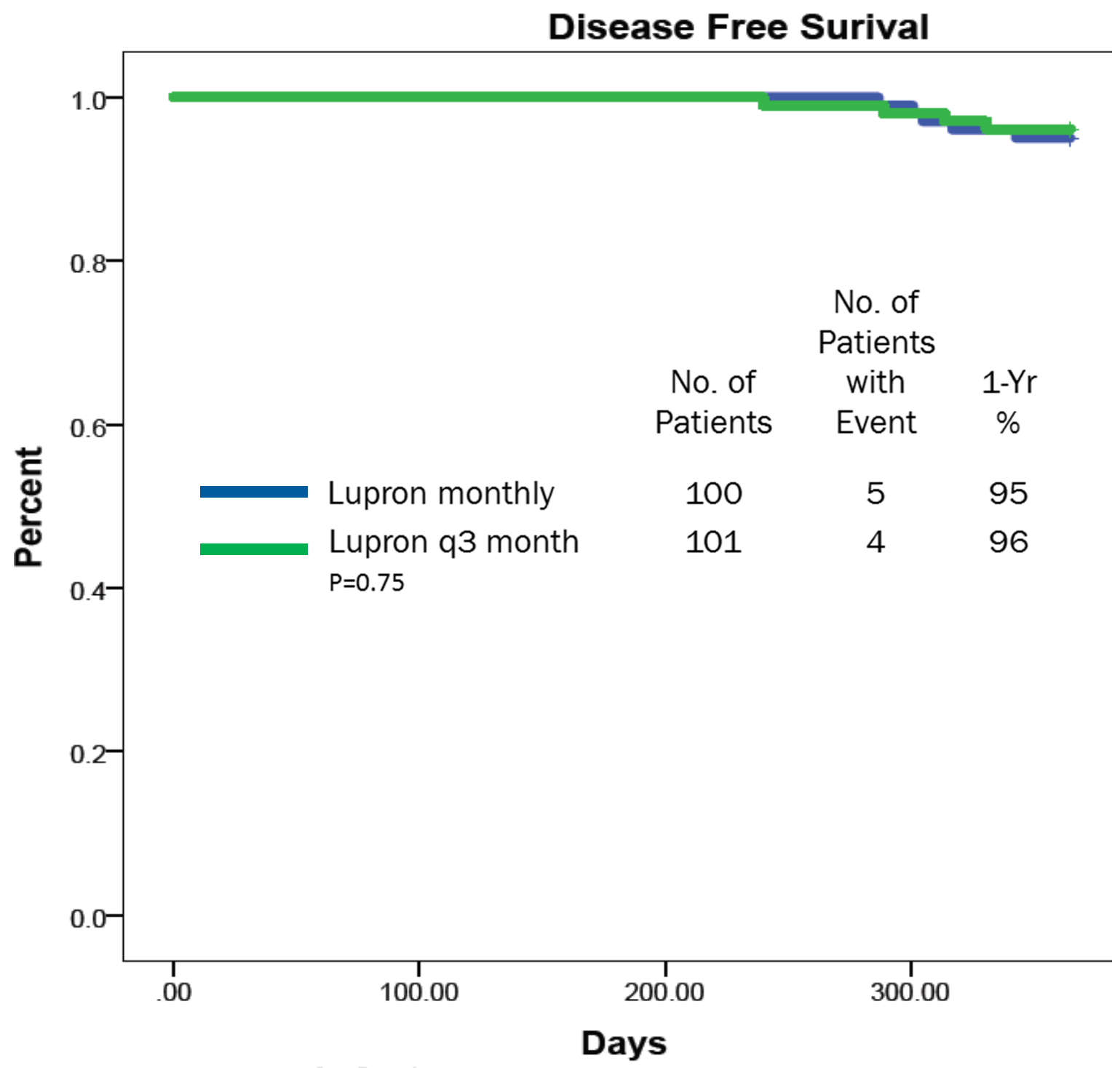


Table 2.

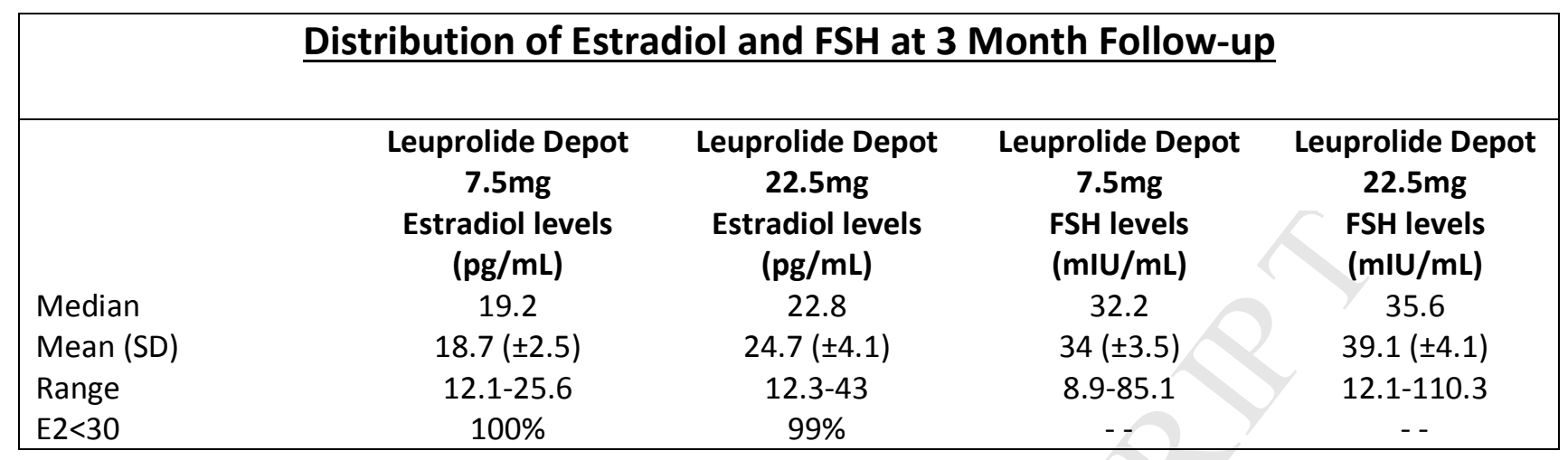


Table 3.

\section{Adverse Effects}

\begin{tabular}{|c|c|c|c|}
\hline Adverse effect & $\begin{array}{c}\text { Leuprolide Depot } \\
\text { 7.5mg } \\
\mathrm{N}=100(\%)\end{array}$ & $\begin{array}{l}\text { Leuprolide Depot } \\
\text { 22.5mg } \\
\mathrm{N}=101(\%)\end{array}$ & $P$ value \\
\hline Hot flashes & $83(83)$ & 87 (86.1) & 0.54 \\
\hline Depression & $46(46)$ & 45 (44.6) & 0.84 \\
\hline Insomnia & $56(56)$ & $53(52.5)$ & 0.51 \\
\hline Fatigue & $68(68)$ & $66(65.3)$ & 0.69 \\
\hline Hypertension & $26(26)$ & $23(22.8)$ & 0.59 \\
\hline Musculo-skeletal & $92(92)$ & $90(89.1)$ & 0.48 \\
\hline Vaginal dryness & $46(46)$ & 49 (48.5) & 0.72 \\
\hline Nausea & $30(30)$ & $27(26.7)$ & 0.61 \\
\hline
\end{tabular}

